\title{
Endobronchial Ultrasound Combined with Clinical Data for Predicting Malignant Peripheral Pulmonary Lesions
}

This article was published in the following Dove Press journal: Cancer Management and Research

\author{
Hong-Yan Ren' \\ Xiao-Ju Zhang' \\ Kun Zhang ${ }^{2}$ \\ Tian-Xiao $\mathrm{Li}^{2}$ \\ Bu-Lang $\mathrm{Gao}^{2}$ \\ Zheng-Xian Chen ${ }^{3}$
}

'Department of Respiratory and Critical Care Medicine, Henan Provincial People's Hospital, Zhengzhou University, Zhengzhou, Henan Province, People's Republic of China; ${ }^{2}$ Department of Interventional Therapy, Henan Provincial People's Hospital, Zhengzhou University, Zhengzhou, Henan Province, People's Republic of China; ${ }^{3}$ Department of Respiratory Medicine, Zhongshan University Sixth Affiliated Hospital, Guangzhou, Guangdong, People's Republic of China
Correspondence: Tian-Xiao Li Tel +861598183691I

Email syylitianxiao@I26.com
Introduction: This study was to develop a simple model for predicting malignancy of peripheral pulmonary lesions (PPLs) based on endobronchial ultrasonography (EBUS) and clinical findings. Methods: Patients who had EBUS for PPLs were analyzed and compared on the EBUS imaging characteristics and clinical data. The malignancy prediction model was established by the logistic equation of probability of malignant PPL based on the data of 135 patients. The model was tested on an additional 50 patients for efficiency.

Results: Among 135 prospectively enrolled patients, 77 (57\%) patients had malignant and 58 (43\%) had benign lesions with the size of $36.5 \pm 19.9 \mathrm{~mm}$. Univariate analysis demonstrated a significant $(P<0.05)$ difference in the serum CEA (borderline $15 \mu \mathrm{g} / \mathrm{mL}$ ) and smoking history between malignant and benign lesions but a non-significant $(P>0.05)$ difference in age (50 years as the cutoff value) and history of extra-thoracic malignancies. Logistic analysis of multiple factors showed that smoking history, serum CEA, borderline, air bronchogram, heterogeneous echo, and anechoic areas were significant $(P<0.02)$ risk factors for malignant lesions. The malignancy prediction model was established by the logistic equation of probability of malignant PPL $(\mathrm{P})=1 /\left[1+\mathrm{e}^{-Z}\right]$, where $\mathrm{Z}=-2.986+1.993 \mathrm{X}_{1}+2.293 \mathrm{X}_{2}+1.552 \mathrm{X}_{3}+1.616 \mathrm{X}_{4}-2.011 \mathrm{X}_{5}+1.718 \mathrm{X}_{6}$, e is the base of the natural logarithm, $\mathrm{X}_{1}$ is the smoking history, $\mathrm{X}_{2}$ is the serum CEA, $\mathrm{X}_{3}$ is the borderline, $\mathrm{X}_{4}$ is the heterogenicity, $X_{5}$ is the air bronchogram, and $X_{6}$ is the anechoic area. The receiver operating characteristic curve had an area under the curve (AUC) of 0.926 ( $95 \%$ confidence interval: $0.883-0.969)$. The sensitivity, specificity, and accuracy were $88.2 \%(30 / 34), 75.0 \%$ $(12 / 16)$, and $92.0 \%(46 / 50)$, respectively, for the logistic equation to predict the malignancy. Conclusion: Endobronchial ultrasonography is a safe and practical method, and the model combining EBUS and clinical data can accurately predict the malignancy of peripheral pulmonary lesions.

Keywords: endobronchial ultrasonography, peripheral pulmonary lesions, malignancy, benign, prediction model

\section{Introduction}

Peripheral pulmonary lesions (PPLs) indicate focal radiographic lesions of opacity with the characteristics of nodules $(\leq 3 \mathrm{~cm})$ or masses $(>3 \mathrm{~cm}) .{ }^{1}$ When pulmonary lobectomy is proposed by some guidelines in patients suffering from PPLs with a high risk of malignancy, ${ }^{2,3}$ computed tomography (CT) screening research has demonstrated that $18 \%-34 \%$ of such surgical procedures are conducted in patients harboring benign nodules. ${ }^{4,5}$ Consequently, correct diagnosis with minimal invasion is strongly favored. 
High-frequency ultrasonic examination with a small probe inside a flexible bronchoscope is termed endobronchial ultrasonography (EBUS). As an evolutional technology invented in the early 1990s, it can enhance diagnostic bronchoscopy by permitting the bronchoscopist to check the peribronchial structures and other peripheral pulmonary nodules. ${ }^{6,7}$ Development of small-diameter ultrasonographic probes has increased clinical application of ultrasonography to tracheal, bronchial, and even peripheral lung lesions in many centers for determining the invasive area of the tumor along the tracheobronchial tree, ${ }^{8}$ understanding relationships in location between lung lesions, lymph nodes, and vessels, ${ }^{9,10}$ capacitating transbronchial needle aspiration guided by EBUS for peribronchial or paratracheal lymph nodes, ${ }^{11}$ evaluating peripheral diseases inside the lung, ${ }^{12}$ appraising appropriate treatment for pulmonary cancer, ${ }^{13,14}$ and locating PPLs to raise the diagnostic accuracy of transbronchial biopsy. ${ }^{15}$

Besides the role in increasing the diagnostic rate of transbronchial biopsy for peripheral pulmonary nodules, the images of EBUS also offer valuable information for determining the nature of peripheral lesions, including tumor, atelectasis, and infection according to different ultrasound features. $^{12,16,17}$ EBUS has enabled physicians to detect lesions from inside the lung and obtain more information besides that obtained via radiology. Kurimoto et $\mathrm{al}^{12}$ have correlated histopathological outcomes from surgicallyexcised specimens to develop a three-type (six-subtype) classification system of ultrasound images for distinguishing malignant from benign lesions, with an elevated consistency between the EBUS images and histopathological findings. However, research concerning these aspects is scarce. The clinical value and application of those characteristics of the EBUS images deserve further studies for confirmation. No study has analyzed the role of EBUS in improving the test probability in differentiating malignancy from benign pulmonary lesions. This study aimed to develop a simple predicting model for judging the nature of PPLs according to images of EBUS and clinical findings.

\section{Patients and Methods}

The ethics committee of Henan Provincial People's Hospital, Zhengzhou University approved this study, with signed informed consent obtained from all participants who were prospectively consecutively enrolled. All procedures performed in the study involving human participants were in accordance with the ethical standards of the institutional and/ or national research committee and with the 1964 Helsinki
Declaration and its later amendments or comparable ethical standards. ${ }^{18}$ Among 150 patients who had computed tomography (CT) and EBUS of lungs for PPLs between September 2010 and September 2015, 135 patients who had pathological confirmation of PPLs were prospectively enrolled for developing the simple prediction model to judge the nature of PPLs, including 84 males and 51 females aged 25-70 years (mean 55.5) (Table 1). Fifteen patients were excluded because it was difficult to obtain a specimen for biopsy due to vessels wrapping the lesion, or because the patient had given up further treatment or was lost to followup. The diagnosis of these patients was eventually made by pathological examination through biopsy, resected sample of lesions, or long-term follow-up observation. Moreover, another cohort of 50 patients with confirmed PPLs between October 2016 and September 2017 were also retrospectively enrolled for testing the efficiency of the prediction model in judging the nature of PPLs based on images and clinical data (Table 2), including 31 males and 19 females with an age

Table I Clinical and Histopathologic Data of Patients in the modelling group (Mean \pm Standard Deviation)

\begin{tabular}{|l|l|}
\hline Variables & Number \\
\hline Sex & \\
Male & 84 \\
Female & 51 \\
\hline Mean age, yr & $55.5 \pm 16.0$ \\
Tumor size, cm & $36.5 \pm 19.9$ \\
Total EBUS duration, min & $4.3 \pm 1.9$ \\
\hline Neoplasm & 77 \\
Adenocarcinoma & 42 \\
Squamous cell carcinoma & 24 \\
Small cell carcinoma & 5 \\
\hline Adenosquamous carcinoma & 1 \\
Bronchioalveolar carcinoma & 1 \\
Non-small cell cancer & 2 \\
Others & 2 \\
\hline Non-neoplasm & 58 \\
Pneumonia & 16 \\
Pulmonary tuberculosis & 11 \\
Lung abscess & 7 \\
Inflammatory pseudotumor & 3 \\
Pulmonary fibrosis and infection & 4 \\
Chondromatous hamartoma of lung & 3 \\
Fungus infection & 5 \\
Pulmonary cyst & 6 \\
\hline
\end{tabular}

Abbreviation: EBUS, endobronchial ultrasonography. 
Table 2 Clinical and Histopathologic Data of Patients in the validation group (Mean \pm Standard Deviation)

\begin{tabular}{|l|l|}
\hline Variables & Number \\
\hline Sex & \\
Male & 31 \\
\hline Female & 19 \\
\hline Mean age, yr & $61.2 \pm 18.0$ \\
Tumor size, cm & $33.20 \pm 16.55$ \\
Total EBUS duration, min & $4.1 \pm 1.8$ \\
Carcinoembryonic antigen & $14.7 \pm 8.09$ \\
\hline Neoplasm & 34 \\
Adenocarcinoma & 20 \\
Squamous cell carcinoma & 9 \\
Small cell carcinoma & 1 \\
Infiltrating adenocarcinoma & 2 \\
Micro-infiltrating adenocarcinoma & 1 \\
Adenocarcinoma in situ & 1 \\
\hline Non-neoplasm & 16 \\
Pneumonia & 9 \\
Pulmonary tuberculosis & 2 \\
Lung abscess & 3 \\
Aspergilloma & 2 \\
\hline
\end{tabular}

Abbreviation: EBUS, endobronchial ultrasonography.

range of 37-76 years (mean 61.2 \pm 18.0 ). The nature of the PPLs in these patients was confirmed by surgical specimen or long-term follow-up observation, and the lesions included were primary lung cancers excluding metastasis at screening. Clinical data of all the patients were collected including sex, age, smoking history, history of extra-thoracic malignancies, and serum carcinoembryonic antigen (CEA) (Tables 1 and 2 ). If the age of the patient was $<50$ years, it was recorded as 0 ; if the age was $\geq 50$ years, it was recorded as 1 . Smoking was recorded as 1 and non-smoking as 0 . Malignant tumor outside the thoracic was recorded as 1 ; otherwise, it was recorded as 0 . If the serum CEA within 1 week was $\geq 5 \mathrm{ng} /$ $\mathrm{mL}$, it was recorded as 1 ; otherwise, it was recorded as 0 .

All enrolled patients received video bronchoscopic examinations (BF-1T260 or BF-1T240; Olympus, Tokyo, Japan) and EBUS. EBUS was conducted using an endoscopic ultrasound system (EUM2000; Olympus, Tokyo, Japan). A 20-MHz miniature radial ultrasonic probe (UMBS20-26R; Olympus, Tokyo, Japan) and an ultrasound Unit (Endoscopic Ultrasound System; Olympus, Tokyo, Japan) were used in the evaluation procedure.

After the pulmonary lesions were localized with chest X-ray radiograph or CT scan, all patients underwent bronchoscopy in a supine position. An anesthetic solution of 2\% Xylocaine was used for local anesthesia. Heart rate and oxygen saturation were monitored by a pulse oximeter during the procedure. After routine video bronchoscopic examination, the miniature probe was filled with physiological saline before insertion into the bronchi with lesions. The probe was introduced up to the point when the operator felt resistance to further advance the probe and, then, the probe was withdrawn slowly for scanning. The shape, size, boundary (clear and continuous), air bronchogram, concentric circle, and internal echo of the lesions were recorded. If the lesion was clear in boundary, round or oval, with air bronchogram, heteroechogenicity, or irregular anechoic areas, it was recorded as 1 . Otherwise, it was recorded as 0 . These variables were chosen based on the imaging and clinical characteristics of the PPLs as indicated in previous studies. ${ }^{8,12,14,17}$ Then, EBUS-guided transbronchial needle aspiration or CT-guided biopsy was performed for histopathological diagnosis. Both EBUS-guided aspiration and CT-guided biopsy were established methods in our hospital. CT-guided biopsy was performed if the pathological diagnosis from EBUS-guided aspiration was different from the clinical presentations or treatment outcomes.

\section{Statistical Analyses}

Statistical analysis was performed with the SPSS software package (version 17.0; IBM, Chicago, IL, USA). Continuous data were presented as mean \pm standard deviation (SD) and were tested with a paired $t$ test. Categorical data were presented as frequency and tested with a chi-square test. The prediction model was identified with binary logistic regression analysis. A forward-conditional method was used to identify variables significant for predicting malignant lesions. The performance of the prediction model was evaluated by calculating the prediction accuracy, the sensitivity and specificity, the receiver operating characteristic (ROC) curve, and the area under the curve. A $P$ value less than 0.05 was considered statistically significant.

\section{Results}

Among the 135 patients for developing the prediction model, 77 (57\%) patients had malignant lesions while $58(43 \%)$ had benign ones (Table 1). The mean size of lesions was 36.5 $\pm 19.9 \mathrm{~mm}$. The major histopathological diagnoses for malignant lesions were adenocarcinoma $(n=42)$, squamous cell carcinoma $(\mathrm{n}=24)$, small cell carcinoma $(\mathrm{n}=5)$, and non-small cell cancer $(\mathrm{n}=2)$ (Figure 1). Benign lesions included pneumonia ( $\mathrm{n}=16)$, tuberculosis $(\mathrm{n}=11)$, lung abscess $(\mathrm{n}=7)$, inflammatory pseudotumor $(n=3$, Figure 2$)$, pulmonary 

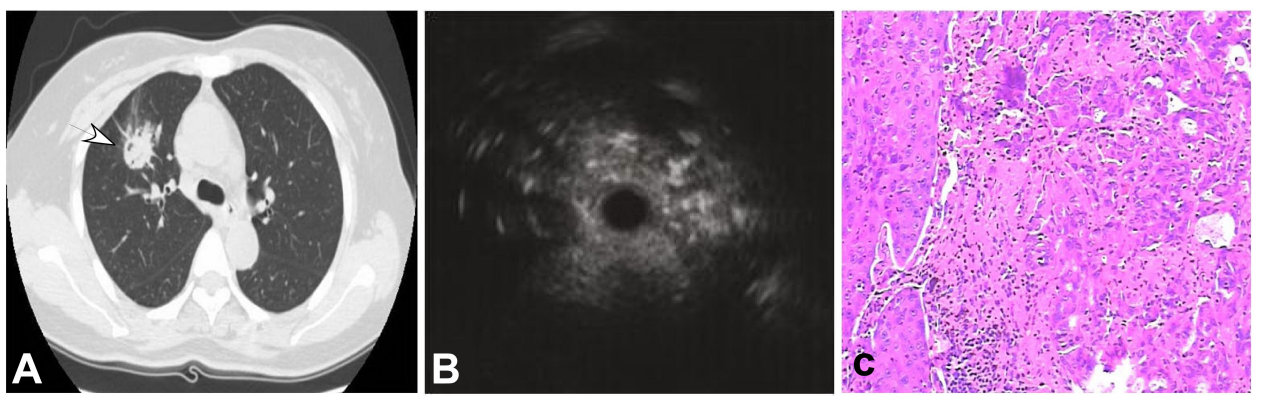

Figure I A 50-year-old man had a peripheral pulmonary lesion in the right lung. (A) A round lesion (arrow) could be seen in the right middle lobe with a size of $9 \mathrm{~mm} \times 9 \mathrm{~mm}$. (B) Endobronchial ultrasonography revealed a heterogeneous lesion with hyper- and hypoechoic areas. (C) Tumor and normal cells existed in the same area with necrosis and fibrosis in the middle $(\mathrm{HE} \times \mid 00)$.

fibrosis and infection $(\mathrm{n}=4)$, pulmonary chondromatous hamartoma $(n=3)$, fungus infection $(n=5)$, and pulmonary cyst $(n=3)$. Among the 50 patients for testing the efficiency of the prediction model, $34(68 \%)$ patients had malignant lesions while the rest $16(32 \%)$ had benign ones (Table 2$)$. The mean size of tumor was $33.20 \pm 16.55 \mathrm{~mm}$. Malignant tumors were classified into the following categories based on the pathological diagnosis: adenocarcinoma $(n=20)$, squamous cell carcinoma $(n=9)$, small cell carcinoma $(n=1)$, infiltrating adenocarcinoma $(\mathrm{n}=2)$, micro-infiltrating adenocarcinoma $(\mathrm{n}=1)$, and adenocarcinoma in situ $(\mathrm{n}=1)$. Benign lesions were pneumonia $(\mathrm{n}=9)$, pulmonary tuberculosis $(\mathrm{n}=2)$, lung abscess $(\mathrm{n}=3)$, and Aspergilloma $(\mathrm{n}=2)$.

In the 135 patients, 8 (4.3\%) cases had adverse reactions during EBUS operations, mainly including minor pulmonary hemorrhage ( 3 cases), severe cough (3 cases), and chest pain ( 2 cases), all of which could be relieved after treatment. The amount of bleeding was $<10 \mathrm{~mL}$ in all 3 cases and was stopped after local lavage treatment with ice saline, application of 1:20,000 epinephrine or thrombin. Cough in 3 patients was relieved after strengthening local anesthesia. Chest pain during probe exploration was relieved after withdrawing the probe.
Univariate analysis of the serum CEA, smoking history, size of lesions, age, and history of extra-thoracic malignancies demonstrated a significant $(P<0.05)$ difference in the serum CEA (borderline $15 \mu \mathrm{g} / \mathrm{mL}$ ) and smoking history in differentiating malignant from benign lesions of PPLs but a non-significant $(P>0.05)$ difference in size of lesions, age (50 years cut-off value), and history of extra-thoracic malignancies. Logistic analysis of multiple factors showed that smoking history, serum CEA, borderline, air bronchogram, heterogeneous echo, and anechoic areas were significant $(P<0.02)$ risk factors for malignant lesions (Tables 3-4).

The malignancy prediction model was established by the following logistic equation:

$$
\begin{aligned}
& \text { Probability of malignant PPL }(\mathrm{P})=1 /\left[1+\mathrm{e}^{-\mathrm{Z}}\right] \\
\mathrm{Z}= & -2.986+1.993 \mathrm{X}_{1}+2.293 \mathrm{X}_{2}+1.552 \mathrm{X}_{3}+1.616 \mathrm{X}_{4} \\
& -2.011 \mathrm{X}_{5}+1.718 \mathrm{X}_{6}
\end{aligned}
$$

where $\mathrm{e}$ is the base of the natural logarithm, $\mathrm{X}_{1}$ is the smoking history, $\mathrm{X}_{2}$ is the serum CEA, $\mathrm{X}_{3}$ is the borderline,
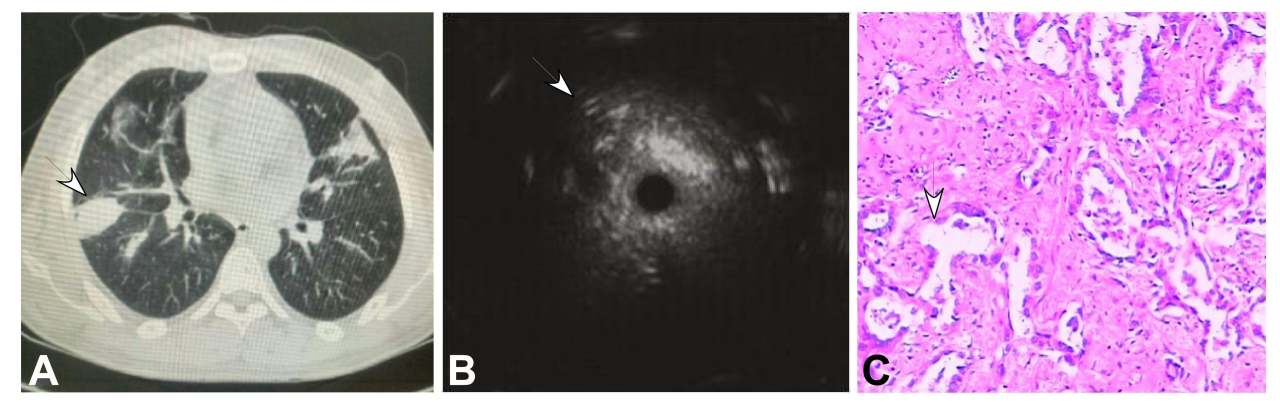

Figure 2 A 65-year-old woman had a peripheral pulmonary lesion (inflammatory pseudotumor) in the lung back segment. (A) A lesion of $30 \mathrm{~mm} \times 28 \mathrm{~mm}$ (arrow) could be seen in the back segment with an air bronchogram. (B) Endobronchial ultrasound could detect a heterogeneous lesion with diffuse short signals. (C) A few distorted and expanded bronchia existed in the inflammatory cells and normal cells $(\mathrm{HE} \times 100)$. 
Table 3 Comparisons of Clinical Factors and EBUS Image Patterns in Predicting Malignancy in Patients

\begin{tabular}{|c|c|c|}
\hline Variables & Neoplasm & Non-Neoplasm \\
\hline \multicolumn{3}{|l|}{ Age } \\
\hline$<50$ & 59 & 51 \\
\hline$\geq 50$ & 18 & 7 \\
\hline \multicolumn{3}{|l|}{ Current or former smoker } \\
\hline Yes & 52 & 20 \\
\hline No & 25 & 38 \\
\hline \multicolumn{3}{|l|}{ Cancer history } \\
\hline Yes & 5 & I \\
\hline No & 72 & 57 \\
\hline Serum CEA & 59 & 51 \\
\hline$<15$ & 15 & 27 \\
\hline$\geq 15$ & 62 & 31 \\
\hline \multicolumn{3}{|l|}{ Lesion configuration } \\
\hline Circular or oval & 38 & 26 \\
\hline Irregular & 39 & 32 \\
\hline \multicolumn{3}{|l|}{ Continuous hyperechoic margin } \\
\hline Yes & 21 & 12 \\
\hline No & 56 & 46 \\
\hline \multicolumn{3}{|l|}{ Distinct borderline } \\
\hline Yes & 45 & 18 \\
\hline No & 32 & 40 \\
\hline \multicolumn{3}{|l|}{ Echo density } \\
\hline Homogenicity & 19 & 36 \\
\hline Heterogenicity & 58 & 22 \\
\hline \multicolumn{3}{|l|}{ Anechoic areas } \\
\hline Yes & 21 & 4 \\
\hline No & 56 & 54 \\
\hline \multicolumn{3}{|l|}{ Air bronchogram } \\
\hline Yes & 25 & 38 \\
\hline No & 30 & 4 \\
\hline \multicolumn{3}{|l|}{ Concentric circle } \\
\hline Yes & 8 & 18 \\
\hline No & 69 & 40 \\
\hline \multicolumn{3}{|l|}{ Internal vessels } \\
\hline Yes & 41 & 35 \\
\hline No & 36 & 23 \\
\hline
\end{tabular}

Abbreviations: EBUS, endobronchial ultrasonography; CEA, carcinoembryonic antigen.

$\mathrm{X}_{4}$ is the heterogenicity, $\mathrm{X}_{5}$ is the air bronchogram, and $\mathrm{X}_{6}$ is the anechoic area.

This logistic equation was tested in predicting the probability of malignant PPLs in 50 patients in comparison with the pathological results (Table 5). The ROC curve is shown in Figure 3 with the area under the curve (AUC) of 0.926 (95\% confidence interval: 0.883-0.969). The $P$ for malignant PPLs was set at $\geq 0.62$ as the cut-off value, and the sensitivity, specificity, and accuracy were $88.2 \%$ (30/34), 75.0\% (12/16), and $92.0 \%$, respectively, for the logistic equation to predict the malignancy.

\section{Discussion}

With wide application and fast development of medical imaging, an increasing number of PPLs have been found, and in individual PPLs, the focus is how to make an early correct diagnosis so as to excise the curable malignant lesions and avoid excessive medical care for benign lesions. Long-term follow-up (for small peripheral nodules), percutaneous image-guided biopsy, PET/CT examination, open lung biopsy, and video-assisted thoracoscopy are multiple approaches to definite diagnosis for PPLs. In 2013, the American College of Chest Physicians (ACCP) pointed out in evidence-based clinical practice guidelines that patients with pulmonary nodules should be assessed and managed by estimating the malignancy probability, performing imaging examinations to better define the lesions, evaluating the risks associated with various management approaches, and eliciting their preferences for management, ${ }^{3}$ with the emphasis on the importance of estimating the probability of malignancy. Radiological examinations such as CT can detect PPLs, but cannot distinguish benign from malignancy, especially for small peripheral nodules. Transcutaneous ultrasound was limited to lesions close to the chest wall. EBUS is an evolving technology, equipped with a $20-\mathrm{MHz}$ mechanical radial probe with an external diameter of $2.0-2.5 \mathrm{~mm}$ and can be easily inserted through a 2.8-mm working channel of a flexible bronchoscope which is flexible enough to review subpleural regions. The EBUS highfrequency probe makes it possible to observe the lesion internal structure, blood vessels, tiny bronchi, and capsular space in the surrounding area. EBUS can provide characteristic information to differentiate the nature of a PPL between neoplasm and non-neoplasm. ${ }^{19}$ In 2007 , Kuo et al $^{17}$ published the positive predictive value in the presence of any two of three malignant features and the excellent negative predictive value if a lesion exhibited none of the three malignant features. But these values are limited to two or three features with some valuable ones possibly missing. After investigation of nine EBUS characteristics and four clinical factors strongly associated with lung cancer in our study, we eventually established 
Table 4 Logistic Model for Predicting Peripheral Lung Cancer Using EBUS and Clinical Features

\begin{tabular}{|l|l|l|l|l|}
\hline Variables & Regression Coefficient (B) & $\boldsymbol{P}$ & Dominance Ratio & 95\% Confidence Interval \\
\hline Constant & -2.986 & 0.000 & 0.051 & \\
CEA & 2.293 & 0.000 & 9.908 & $2.96-33.09$ \\
Smoking history & 1.993 & 0.000 & 7.338 & $2.42-22.21$ \\
Distinct borderline & 1.552 & 0.003 & 4.723 & $1.71-13.05$ \\
Heterogeneous echo & 1.616 & 0.002 & 5.033 & $1.82-13.93$ \\
No air bronchogram & -2.011 & 0.000 & 7.463 & $2.56-20.00$ \\
Anechoic areas & 1.748 & 0.029 & 5.745 & $1.20-27.54$ \\
\hline
\end{tabular}

Abbreviations: CEA, carcinoembryonic antigen; EBUS, endobronchial ultrasonography.

Table 5 Testing of the Logistic Equation with Pathological Results in 50 Patients

\begin{tabular}{|l|l|l|l|}
\hline \multirow{2}{*}{ Logistic Equation } & \multicolumn{2}{|l|}{ Pathological Results } & \multirow{2}{*}{ Total } \\
\cline { 2 - 3 } & Malignant & Benign & \\
\hline Malignant & 30 & 12 & 42 \\
Benign & 4 & 4 & 8 \\
Total & 34 & 16 & 50 \\
\hline
\end{tabular}

the prediction model for evaluating the probability of malignancy.

The history of disease and clinical presentations are important in differentiating benign from malignant PPLs. For the diagnosis of benign lesions, the pathology of biopsy tissue and lavage fluid and culture of the lavage

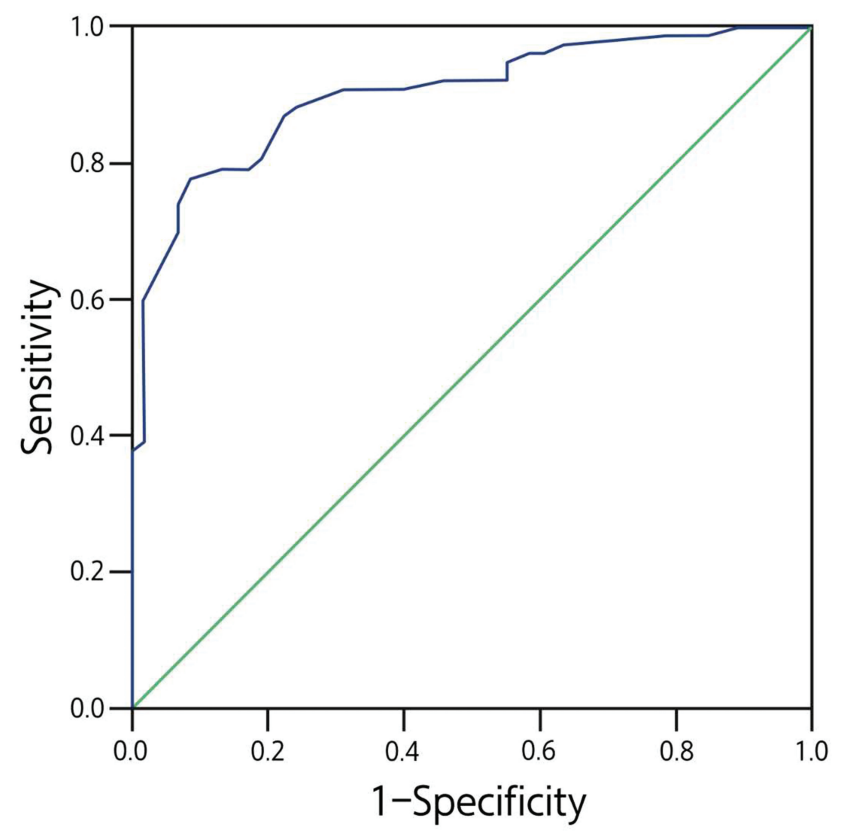

Figure 3 Receiver operating characteristic curve for the direction model for diagnosis of malignant peripheral pulmonary lesions (PPLs). The prediction model was developed based on data from 135 consecutive patients. fluid are helpful in getting the correct diagnosis. For patients with benign lesions, a correct diagnosis may be reached by following up the patient for a certain period of time until the symptoms of the patient are relieved or the signs of the lesion become definite. Our study found that smoking history and tumor marker CEA can be used as independent factors for predicting benign from malignant PPLs and that smoking is a risk prediction factor for lung cancer. The use of CEA as a prognostic and predictive marker in patients with lung cancer was once widely debated. In a review of CEA as a tumor marker in lung cancer by Grunnet and Sorensen published in $2012,{ }^{20}$ it was found that the serum level of CEA carries prognostic and predictive information of risk of recurrence and of death in non-small cell lung cancers independent of treatment or study design. However, age and extra-thoracic tumors were not found in our study to have a significant difference $(P>0.05)$ in differentiating benign from malignant PPLs probably because of fewer patients or the definition of age division of 50 years in our study. Moreover, the size of lesion was also eliminated in the model because initial univariate analysis did not find it to be a significant factor.

In our study, nearly $70 \%$ of neoplasms were round or oval, whereas almost $80 \%$ of non-neoplasm lesions were irregular. The tumor masses grow in a centrally expansive manner and will demonstrate a round or oval shape when they are small and restrained by surrounding tissues. They will eventually demonstrate malignant invasive nature with lobulated margins when they grow big enough. Therefore, most of the small PPLs of malignancy were round and oval. However, unlike the small PPLs of malignancy, pneumonia is not round nor oval and the infection can distribute through the bronchioles. With infection transmission, pneumonia expands at different speeds because of different resistance from interstitial substance and alveolar and shows signs of "wave-shape", "ladder-shape", "sawtooth", and "star 
shape". ${ }^{16}$ The neoplasm lesions generally grow in an expansive, compressive, or padding manner, or along the alveolar wall, with the surrounding tissues being compressed into a thin layer of lung atrophy. Moreover, the pulmonary nodules and nearby aerated lung tissues have different tissue components, capacitating the peripheral lesions to be more hypoechoic than the surrounding tissues. Under ultrasonographic examination, peripheral lung cancers exhibit a distinct margin.

The patterns of internal echogenicity were classified as "homogenicity" or "heterogenicity". Homogeneity refers to the consistency of the content, size, and distribution of the lesion internal echo area. Images of homogeneous internal echo may consist of exudates and cell debris within the alveoli in non-neoplasm patients. The heterogeneous echoes demonstrated a mosaic pattern in the distribution of imaging particles with varied sizes of the particles. This EBUS echo feature is closely related to cell arrangement and amount of fibrous stroma. Previous studies $^{6,16}$ have demonstrated that a heterogeneous echogenicity was probably a malignant lesion. In lung cancers, tumor cells grow rapidly to replace normal alveolar lining cells. Consequently, the loss of normal tissues combined with chaotic tumor cell growth including necrosis, central fibrosis, and hemorrhage enables the lung lesion to have a heterogeneous feature on the EBUS images.

The air bronchogram in EBUS images is featured by laminar and regular hyperechoic short lines aligned concentrically in a hypoechoic background, which reflects wellpreserved anatomical structures of bronchioles and airspace in the consolidative pulmonary parenchyma. Benign lesions often present congestion, edema, necrosis, and hyperplasia, containing distorted or dilated bronchi filled with air, which are reflected in the EBUS images as dots or short-discrete hyperechoic signs. Thus, a lesion with the air bronchogram distributed on the outside nearly in concentric circles under EBUS examination is probably benign in nature. However, cartilage and calcification should be excluded. Kurimoto et $\mathrm{al}^{12}$ reported that no air bronchogram was a characteristic of poorly differentiated adenocarcinoma and small cell cancer. But in our study, this sign can be detected in various pathological lesions.

In our study, 25 cases had anechoic areas, of which 21 (84\%) were diagnosed as a neoplasm. When the blood supply is not sufficient for fast-growing tumors, the tumor may have liquefactive necrosis which presents as anechoic areas on ultrasound. Therefore, the appearance of this sign probably indicates malignancy, consistent with a previous report. ${ }^{12}$
Tumor masses may often present with hypoechogenicity in the internal structure; however, central necrosis and condensation can present anechoic areas on the EBUS images.

EBUS is an emerging technique, and the model of EBUS combined with clinical presentations has a sensitivity of $88.2 \%$ and specificity of $75.0 \%$ for predicting malignant from benign lesions in our study. The sensitivity is close to that $(74 \%-96 \%)$ with CT-guided biopsy. $^{21}$ This may indicate that comprehensive analyses combining clinical data and imaging features can increase the sensitivity, specificity, and diagnostic accuracy. For the AUC curve, the closer to 1 the AUC, the greater the diagnostic value. The diagnostic value is relatively low at the AUC between 0.5 and 0.7 but high at the AUC between 0.7 and 0.9. In our study, the AUC was 92.6\%, indicating a high diagnostic value of the model.

Some limitations may exist in this study. The small cohort of patients and the Chinese ethnicity were two limitations which should be resolved in future studies.

Although EBUS may provide a new and valuable way to visualize the internal structures of peripheral pulmonary lesions and the model established with EBUS combined with clinical features has very good accuracy, the model is not intended to be used as a stand-alone test, but rather as a tool to help guide the selection and interpretation of subsequent diagnostic tests. Future studies should evaluate the accuracy of this prediction model in a larger cohort or non-Chinese cohort of patients.

In conclusion, EBUS is a safe and practical examination method, and the model combining EBUS and clinical data can accurately predict the malignancy of peripheral pulmonary lesions.

\section{Disclosure}

The authors declared that they have no conflicts of interest in this work.

\section{References}

1. Khan T, Usman Y, Abdo T, Chaudry F, Keddissi JI, Youness HA. Diagnosis and management of peripheral lung nodule. Ann Transl Med. 2019;7:348. doi:10.21037/atm.2019.03.59

2. Barnett J, Belsey J, Tavare AN, et al. Pre-surgical lung biopsy in management of solitary pulmonary nodules: a cost effectiveness analysis. $J$ Med Econ. 2019;22:1307-1311. doi:10.1080/13696998.20 19.1665322

3. Gould MK, Donington J, Lynch WR, et al. Evaluation of individuals with pulmonary nodules: when is it lung cancer? Diagnosis and management of lung cancer, 3rd ed: american college of chest physicians evidence-based clinical practice guidelines. Chest. 2013;143:e93Se120S. doi:10.1378/chest.12-2351 
4. Kaaki S, Kidane B, Srinathan S, Tan L, Buduhan G. Is tissue still the issue? Lobectomy for suspicious lung nodules without confirmation of malignancy. J Surg Oncol. 2018;117:977-984. doi:10.1002/jso.25003

5. Swensen SJ, Jett JR, Hartman TE, et al. Ct screening for lung cancer: five-year prospective experience. Radiology. 2005;235:259-265. doi:10.1148/radiol.2351041662

6. Kho SS, Tie ST. Radial probe endobronchial ultrasound (r-ebus) guided transbronchial cryobiopsy in the diagnosis of peripheral solitary pulmonary nodule. Med J Malaysia. 2019;74:349-351.

7. Yasufuku K, Nakajima T, Chiyo M, Sekine Y, Shibuya K, Fujisawa T. Endobronchial ultrasonography: current status and future directions. J Thorac Oncol. 2007;2:970-979. doi:10.1097/JTO.0b013e318153fd8d

8. Kurimoto N, Murayama M, Yoshioka S, Nishisaka T, Inai K, Dohi K. Assessment of usefulness of endobronchial ultrasonography in determination of depth of tracheobronchial tumor invasion. Chest. 1999;115:1500-1506. doi:10.1378/chest.115.6.1500

9. Gu Y, Shi H, Su C, et al. The role of endobronchial ultrasound elastography in the diagnosis of mediastinal and hilar lymph nodes. Oncotarget. 2017;8:89194-89202. doi:10.18632/oncotarget.19031

10. Nakajima T, Shingyoji M, Anayama T, Kimura H, Yasufuku K, Yoshino I. Spectrum analysis of endobronchial ultrasound radiofrequency of lymph nodes in patients with lung cancer. Chest. 2016;149:1393-1399. doi:10.1016/j.chest.2016.01.015

11. Medford AR. Endobronchial ultrasound-guided transbronchial needle aspiration. Int J Clin Pract. 2010;64:1773-1783. doi:10.1111/j.17421241.2010.02454.x

12. Kurimoto N, Murayama M, Yoshioka S, Nishisaka T. Analysis of the internal structure of peripheral pulmonary lesions using endobronchial ultrasonography. Chest 2002;122:1887-1894. doi:10.1378/ chest.122.6.1887

13. Aziz F. Endobronchial ultrasound-guided transbronchial needle aspiration for staging of lung cancer: A concise review. Transl Lung Cancer Res. 2012;1:208-213. doi:10.3978/j.issn.2218-6751.20 12.09.08
14. Herth F, Becker HD, Manegold C, Drings P. Endobronchial ultrasound (ebus)-assessment of a new diagnostic tool in bronchoscopy for staging of lung cancer. Onkologie. 2001;24:151-154. doi:10.1159/ 000050303

15. Oki M, Saka H, Kitagawa C, Kogure Y, Mori K, Kajikawa S. Endobronchial ultrasound-guided transbronchial biopsy using novel thin bronchoscope for diagnosis of peripheral pulmonary lesions. J Thorac Oncol. 2009;4:1274-1277. doi:10.1097/JTO.0b013e31 $81 \mathrm{~b} 623 \mathrm{e} 1$

16. Huang Y, Chen Z, Ren H, He B, Li X. [differential diagnosis of malignant and benign peripheral pulmonary lesions based on two characteristic echo features of endobronchial ultrasonography]. Nan Fang Yi Ke Da Xue Xue Bao. 2012;32:1016-1019. Chinese.

17. Kuo CH, Lin SM, Chen HC, Chou CL, Yu CT, Kuo HP. Diagnosis of peripheral lung cancer with three echoic features via endobronchial ultrasound. Chest. 2007;132:922-929. doi:10.1378/chest.06-3106

18. Majem M, Juan O, Insa A, et al. Seom clinical guidelines for the treatment of non-small cell lung cancer (2018). Clin Transl Oncol. 2019;21:3-17. doi:10.1007/s12094-018-1978-1

19. Chao TY, Lie Ch Fau - Chung Y-H, Chung Yh Fau - Wang JL, et al. Differentiating peripheral pulmonary lesions based on images of endobronchial ultrasonography.

20. Grunnet M, Sorensen JB. Carcinoembryonic antigen (cea) as tumor marker in lung cancer. Lung Cancer. 2012;76:138-143. doi:10.1016/ j.lungcan.2011.11.012

21. Zhan P, Zhu QQ, Miu YY, et al. Comparison between endobronchial ultrasound-guided transbronchial biopsy and ct-guided transthoracic lung biopsy for the diagnosis of peripheral lung cancer: a systematic review and meta-analysis. Transl Lung Cancer Res. 2017;6:23-34. doi:10.21037/tlcr.2017.01.01

\section{Publish your work in this journal}

Cancer Management and Research is an international, peer-reviewed open access journal focusing on cancer research and the optimal use of preventative and integrated treatment interventions to achieve improved outcomes, enhanced survival and quality of life for the cancer patient.
The manuscript management system is completely online and includes a very quick and fair peer-review system, which is all easy to use. Visit http://www.dovepress.com/testimonials.php to read real quotes from published authors. 